\title{
Envisioning a Wireless Future
}

\author{
Gunasundari R, \\ Department of ECE, Pondicherry Engineering College, Puducherry- 605 014, INDIA \\ Email: r_gunasundari@rediffmail.com
}

When taken in small doses, technical evolution seems fluid and almost natural. But considering the advances in technology over the course of decades reveals a much more radical transformation of the way we live, communicate and entertain. Just the way we communicate with one another has undergone a dramatic shift. Everything from public manners to safety is being challenged by anywhere, anytime communication. This article presents a scenario considering where mobile communications was then and what it looks like today, what might our wireless future look like 20 years hence?

\section{No wires, no hands}

The young generation already finds it hard to believe that their elders used to talk on a phone tied to a wall? The next generation will be equally incredulous that we once had to stick something up to our heads to talk. The common prophecy to this end is implantation. Many visionaries have put forth the notion of embedded intelligence, where simple thought is all that is necessary to telepath "mental speech" wirelessly to another individual anywhere in the world. But, the risks and concerns associated with implantation as even necessary, given that very tiny devices can be lodged in clothing, jewelry or even behind an ear. Those tiny displays on our mobile will be expanded and morphed with our regular vision, possibly via transparent LCD displays built into glasses or even contact lens-like screens. Augmented reality will be a radical shift for society to deal with. Imagine getting hacked or stung by a virus in this medium--it means more than just corrupted information; an alternate reality could invade your world.

\section{One with nature}

Personal wireless communication needs to be implemented via point-to-point technology--not broadcast. We've been living in a broadcast world from day one, when the very first signals were sent sans wires. Looking at the nature of wireless signals, they naturally lend themselves to broadcasting--spewing energy omni directionally for anyone in range to detect and consume. To utilize broadcast signals for secure, personal communications between two parties is inconsistent with the uncontrolled nature of electromagnetic radiation. Smart antennae are moving us in the right direction, where electromagnetic waves are focused on mobile devices as they move, but yet to be standardized. Radical advances in spatial processing dramatically improve the capacity, security and quality of personal wireless communications.

\section{Unfettered access, immediate authentication}

It still takes time to access some audio and visual information or entertainment. But future wireless technology will ensure everything is accessible immediately upon payment for service or transmission of identity. It will speed your way into work, VIP parking areas or any other areas restricted on the basis of identity. Hopefully, it will also do something to improve long lines at government offices and the airport.

It is the need and desire of people to access the Internet from anywhere in the world that is driving many of the new changes that are occurring in the largest consumer market ever the wireless mobile device market. Fig1 represents the mobile device view and there are three main routes to wirelessly access the Internet. Voice is still the main use of mobile devices and the GSM and CDMA networks around the world now connect over 2.3 billion wireless subscribers. The cellular standards are evolving to add high speed data connections, and cellular remains the way we connect from remote locations many miles away from a base station.

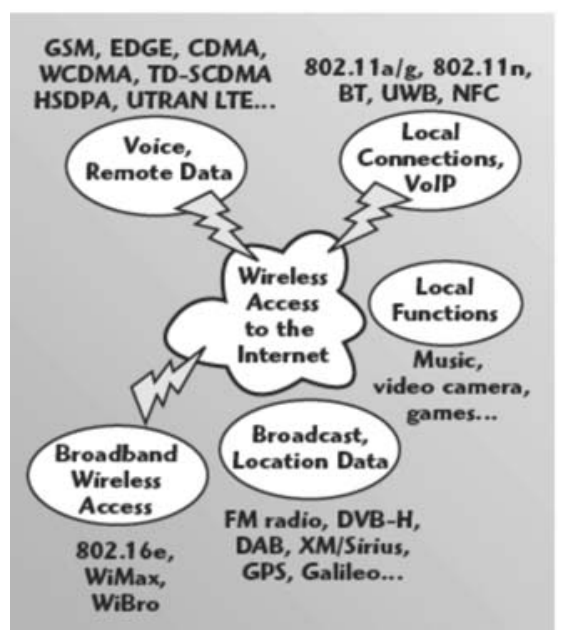

Fig. 1 Wireless Access to the Internet Everywhere and Entertaining Functions will lead to Highly Usable and fun Mobile Devices.

For local connections Bluetooth"is rapidly becoming a common feature in mobile devices with almost 30 
percent attachment rate expected in 2006. This attachment rate is expected to grow to more than 50 percent over the next three years. Today we are seeing other radios being combined with Bluetooth, such as WiFi, FM radio and Near Field Communications (NFC). Soon, there will be a combination Bluetooth and Ultra-wideband (UWB) devices to further enhance the wireless distribution of multimedia content. It will not be long before that $5 \mathrm{MB}$ presentation will be beamed to an UWB-enabled plasma monitor in the conference room or displayed directly in real time onto your colleagues' mobile device at the table.

What's clear about the future 20 years down the road is how integrated into our lives wireless technology will have become. The result will be nearly unbounded connectivity. But the promise of omnipresent wireless communication presents us with an unexpected dilemma. On the one hand, we can look forward to instantaneous access to what we want. Couple that with new challenges to our social norms as well as our traditional expectations of privacy. But that's fodder for a future column.

\section{REFERENCES}

[1] Alastair Upton and Victor Steel, "The Current State of Technology and Future Trends in Wireless Communications and Applications", Microwave Journal, Vol. 49, No. 9, September 2006

[2] Dave Mock," Envisioning a wireless future", Amazon Review, CNET News 2005. 